\title{
Seckel syndrome with cutaneous pigmentary changes: two siblings and a review of the literature
}

\author{
Arzu Kilic ${ }^{1}$, Seray Külcü Çakmak², Timur Tuncali³, Ozlem Koz ${ }^{4}$, Esra Ozhamamci ${ }^{5}$ Oztan Yasun ${ }^{6}$, Ferda Artuz ${ }^{2}$ \\ ${ }^{1}$ Department of Dermatology, Medical Faculty, Balıkesir University, Balıkesir, Turkey \\ Head of the Department: Arzu Kilic \\ ${ }^{2}$ Department of Dermatology, Ankara Numune Education and Research Hospital, Ankara, Turkey \\ Head of the Department: Ferda Artuz \\ ${ }^{3}$ Department of Medical Genetics, School of Medicine, Ankara University, Ankara, Turkey \\ Head of the Department: Hatice llgin Ruhi \\ ${ }^{4}$ Department of Ophthalmology, Ankara Numune Education and Research Hospital, Ankara, Turkey \\ Head of the Department: Yildiz Ozdemir \\ ${ }^{5}$ Department of Pathology, Ankara Numune Education and Research Hospital, Ankara, Turkey \\ Head of the Department: Sezer Kulacoglu \\ ${ }^{6}$ Dentistry Clinic, Ankara Numune Education and Research Hospital, Ankara, Turkey \\ Head of the Department: Erbil Celtek Altinok
}

Seckel syndrome (SCKL) is an extremely rare form of primordial dwarfism characterized by growth delay, proportionate extreme short stature, a prominent beak-like nose, hypoplasia of the malar area, small chin, microcephaly, and skeletal malformations [1-4]. In this review, two siblings with a combination of clinical, skeletal, ocular, dental and cytogenetic findings are presented in view of SCKL.

Case 1: A 26-year-old woman presented to our dermatology outpatient clinic for pigmentary changes on her body that have existed for ten years. She has not approached any doctor due to her symptoms before. On dermatological examination, multiple milimetric hyperand hypo-pigmented macules were detected on her body primarily localized on both axillary regions and both sides of her neck. On physical examination, she was noticed to have extremely short stature and low weight. General appearance of the patient was characterized by a small, narrow face and forehead, slightly beaked nose, midfacial hypoplasia, very stunted stature with microcephaly (Figure 1). She also displayed high-pitched voice. The age of menarche was 15 years. She had regular menstrual cycles.

Severe growth retardation, abnormal facial features and presence of pigmentary skin lesions led us to investigate the patient for a possibility of a syndrome.

The patient was born to a GP1 21-year-old woman after an uneventful pregnancy. Her parents were nonconsanguineous and healthy. She was delivered at full term with normal delivery with birth weight of $1400 \mathrm{~g}$. The family had four female and four male children. An unexamined male sibling has been reported to be underdeveloped and weak, while the other five siblings were defined as healthy.

The patient had developmental difficulties by birth, especially about weight gain.

On general examination, she had an abnormal short stature with a weight of $25 \mathrm{~kg}$ and height of $135 \mathrm{~cm}$. Her head circumference was $48 \mathrm{~cm}$.

On laboratory examinations, results of complete blood count and biochemistry panel were normal except low ionized calcium levels (3.87 mg/dl, normal: 4.64-5.28 mg/dl). Parathormone (PTH) levels (69.1 pg/ml, normal: $15-65$ pg/ $\mathrm{ml}$ ) were high while the levels of vitamin D was quite low $(6.2 \mathrm{ng} / \mathrm{ml}$, normal: $25-80 \mathrm{ng} / \mathrm{ml})$ indicating osteomalacia.

On radiological examination, no pathology was detected on chest radiography. The bone mineral density test showed osteopenia. Osteopenia was detected in all bone radiographic examinations. The detected findings on plain X-ray films were failure of bone tubulation on both mid phalanxes of the $5^{\text {th }}$ fingers of both hands and a bony spur on the surface of the distal tibial metaphysis which were compatible with ostoechondroma and slight scoliosis.

On ophthalmological examination, her visual acuity (Snellen chart) was 0.5 (with $+3.75,+0.7520^{\circ}$ ) in the right eye and 0.3 (with $+4.0,+0.75160^{\circ}$ ) in the left eye.

Address for correspondence: Arzu Kilic, Department of Dermatology, Medical Faculty, Balikesir University, Cagis Yerleskesi, 10100 Balikesir, Turkey, fax: +90 26661210 10, e-mail: kilicarzu@gmail.com Received: 21.01.2015, accepted: 18.02.2015. 
Axial length measurement using IOL master (Carl Zeiss AG, Germany) showed that she had a shorter axial length $(17 \mathrm{~mm})$ in both eyes. She was pseudophakic in both eyes from previous cataract surgery when she was 23 years old. There was no significant retinal abnormality observed. Electroretinography (ERG) showed subnormal amplitude of rod-mediated and cone-mediated responses.

On intraoral examination, the patient had severe periodontitis, and as a result she had multiple loose teeth, bacterial plaques, lack of posterior first molar teeth on superior chin. The anatomical morphology of the teeth was normal, but she had enamel hypoplasia especially on superior and inferior chin on labial surfaces. On radiographic examination, third molar teeth were not observed.

Psychiatric examination revealed borderline mental capacity. She was able to complete her education only until the end of primary school due to her mental capacity and her reduced visual activity.

Skin biopsy was obtained from the hyperpigmented lesions and the histopathological examination revealed basilar hyperpigmentation, melanophages through papillary dermis and minimal perivascular mononuclear cell infiltration.

Because of these findings, her brother with the same features was also invited to our dermatology clinic.

Case 2: The male patient was the brother of the first patient and he was 14 years old. He had also low weight with a short stature.

The patient was born to a GP6 woman after an uneventful pregnancy. He was delivered at full term with birth weight of $1700 \mathrm{~g}$.

The patient also had developmental difficulties by birth, especially with weight gain like her sister. On general examination, he had an abnormal short stature with a weight of $30 \mathrm{~kg}$ and height of $137 \mathrm{~cm}$. His head circumference was $48 \mathrm{~cm}$. He had a receding chin and forehead (Figure 2). No pathology was observed on dermatological examination.

On laboratory examination, results of complete blood count and biochemistry panel were normal except low ionized calcium levels (4.3 mg/dl, normal: 4.64-5.28 mg/dl). The vitamin D level was low while PTH levels were normal.

Osteopenia was detected on all bone radiographic examinations. Chest X-ray, abdominal ultrasonography and cranial magnetic resonance examinations were normal.

On ophthalmological examination, he had a shorter axial length $(19 \mathrm{~mm})$ in both eyes. Anterior and posterior segment findings were normal. The ERG showed subnormal amplitude of rod-mediated and cone-mediated responses. His visual acuity (Snellen chart) was 0.5 (with $\left.+1.50,+0.7520^{\circ}\right)$ in the right eye and $1.0\left(\right.$ with $\left.+0.75170^{\circ}\right)$ in the left eye.

On intraoral examination, dimensions of the chin were under the norms. Dimensional developmental defects of teeth were detected. The arch of the chin was

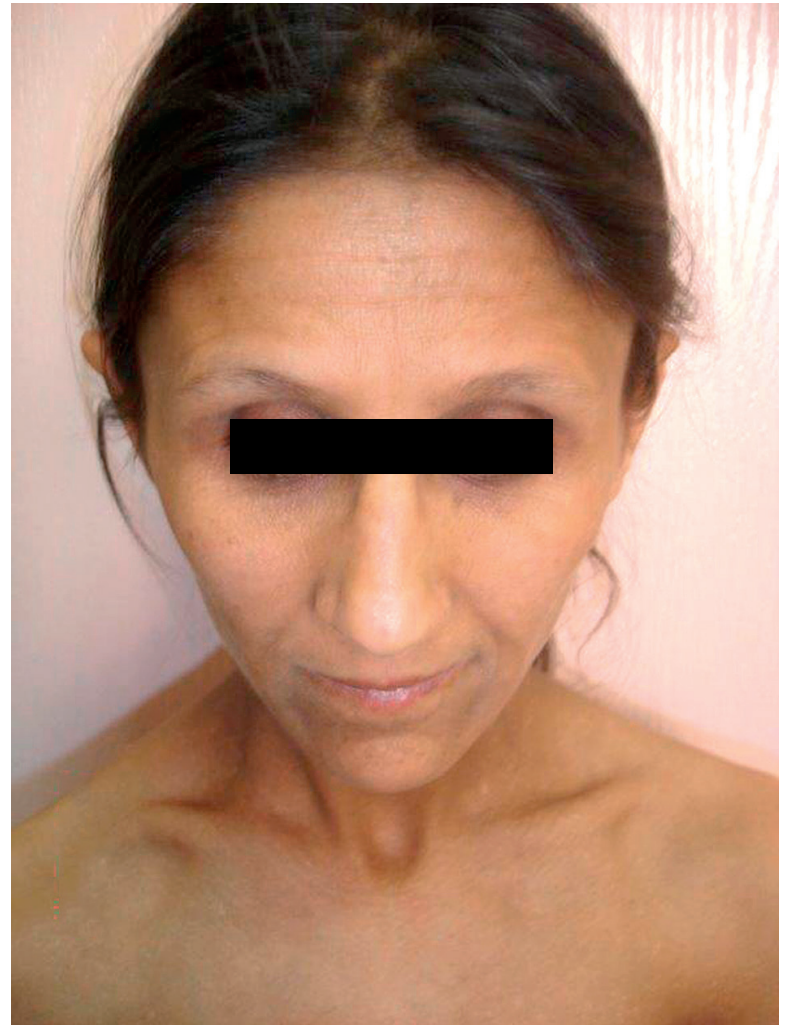

Figure 1. The appearance of milimetric hyper and hypopigmented macules on both sides of her neck

incompetent. Oral hygiene status was poor and multiple dental caries were detected. Third molar teeth were absent.

Both patients were further consulted at a medical genetics department. Consistent clinical findings in both siblings supported the decision to test the patients' chromosomes for instability in response to clastogenic agents which are supposed to be caused by defects in the ATR gene coding for ataxia telangiectasia and Rad3 related protein in at least one type of Seckel syndrome. Peripheral blood samples were obtained from patients and cultured for chromosomal instability testing in lymphocytes. Diepoxybutane (DEB) and mitomycin-C (MMC) were used as clastogenic agents for their DNA cross-linking damage properties with a replicate set of cultures to serve as untreated controls with methods described elsewhere [5]. One hundred metaphases were evaluated for each sample and condition. In addition, an unmatched healthy control subject was also studied with the same methodology. The female patient had $24 \%$ spontaneous breaks from untreated, $72 \%$ from DEB induced and $82 \%$ from MMC induced culture cells. While the male sibling had no spontaneous breaks, he had $10 \%$ of the breaks from the DEB and $86 \%$ of the breaks from the MMC induced culture cells. The matched untreated and control cultures were detected as break free. Both patients were found to 

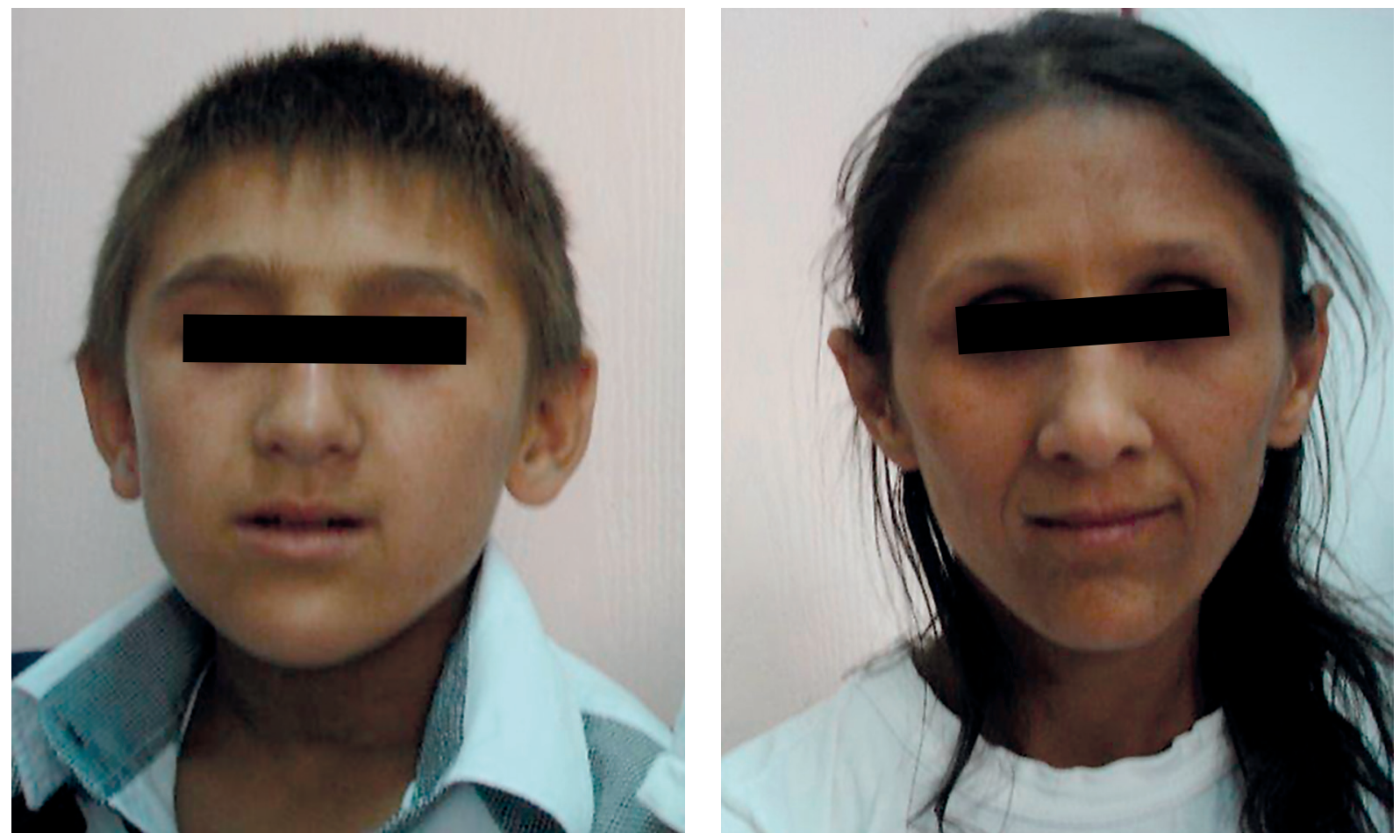

Figure 2. General appearance of patient 1 and 2

have normal chromosomal constitution in conventional G-banded metaphase analyses.

SCKL (OMIM 210600), belonging to the group of osteodysplastic primordial dwarfism, is characterized by antenatal and postnatal growth delay [1-4]. It is a genetically heterogeneous autosomal recessive disorder and has a wide phenotypic heterogeneity between affected patients. Genetic heterogeneity has also been proven with three loci identified to date and suggested that the syndrome was associated with a defective ATR-dependent DNA damage signaling pathway $[4,6]$.

The family tree with two affected sibs of unrelated parents was consistent with autosomal recessive inheritance. The high percentage of chromosome breaks detected in both cases suggested a causal relation with an impaired DNA damage repair mechanism. These results were also in accord with the results of Bobabilla-Morales et al. and others [7].

In addition to the characteristic craniofacial dysmorphism; ocular findings, skeletal defects, mental retardation, cardiovascular, hematopoietic, endocrine, and central nervous system abnormalities have been reported [1, $3,4,6,8]$. The full phenotypic spectrum of this clinically and genetically heterogeneous syndrome is yet to be delineated. Two siblings reported here in this article also demonstrated clinical heterogeneity.

The most common ophthalmological findings are telecanthus, narrow palpebral fissures, and large "bulging eyes" [8-10]. Our female patient had a cataract sur- gery and intraocular lens implantation when she was 23 years old. Similar to our case; a 17-year-old boy with SCKL who had spontaneous bilateral lens dislocation with subcapsular and cortical cataract has been reported [11]. Subnormal amplitude of rod-mediated and conemediated responses in the ERG test is another important finding in both our cases. Severe bilateral pigmentary retinopathy was shown in three patients by Guirgis et al. [9]. Similarly, they reported no detectable rod or cone responses in ERG. It was shown that the photoreceptor nuclei were variably reduced in number and columns were loosely packed or disorganized in the retina in Cenpj $j^{\mathrm{tm} / \mathrm{tm}}$ animals [12]. This photoreceptor anomaly shown in this study might explain subnormal rod and cone mediated responses seen in our cases.

Regen et al. reported a review of dental manifestations associated with SCKL [13]. Dental agenesis, short roots, primary teeth microdontia, oligodontia, enamel hypoplasia, loose teeth have been reported as the most common dental findings [13-15]. Our cases also had prominent dental manifestations.

Various abnormalities of the central nervous system in various degrees have also been reported in the literature [16-21]. Agenesis of corpus callosum, hypoplasia of the cerebellar vermis, a dysgenetic cerebrum with pachygyria, open and closed lip schizencephaly, tonsillar herniation, semilobar holoprosencephaly are the associated findings that have been reported [16-19]. Cranial mag- 
netic resonance images of both our patients showed no abnormalities.

The other rare manifestations of SCKL with the involvement of endocrine, cardiac, vascular, gastrointestinal and hematological systems have also been reported [22-30]. Adiyaman et al. reported a case of SCKL with endocrine abnormalities including premature pubarche, hyperinsulinism, accompanying mild-hypertriglyceridemia, dyslipidemia, and hyperandrogenism [22]. Our female patient had regular menstrual cycles but low calcium and high PTH levels secondary to osteomalacia and vitamin D treatment was started.

Our patients were investigated for cardiovascular and gastrointestinal manifestations and no abnormalities were detected.

Another complication that has been reported in SCKL is acute myeloid leukemia (AML) [31]. Our patients did not have any hematological abnormalities.

Another rare manifestation of SCKL is cutaneous involvement [4, 32]. Brackeen et al. have reported a female patient with erythematous, scaly, lichenified plaques on her hands, knees, and feet with the diagnosis of atopic dermatitis and hypopigmented macules and papules on her chest, back, extremities, and sides of her face [4]. Neither our patient nor her parents described any previous inflammatory skin changes previously. No dermatological findings were detected in the male patient. As suggested in the literature [4, 32], these pigmentary changes are likely to be a part of the syndrome.

In most cases, the diagnosis of SCKL depends on the recognition of typical clinical findings. Our cases also had characteristic findings related to this syndrome such as prenatal onset of marked growth retardation, significant microcephaly, a prominent beaked nose and micrognathia. The patients must be followed up for the presence of hematological abnormalities, including anemia, pancytopenia, and acute myeloid leukemia and also for involvement of other systems including cardiovascular, neurological abnormalities. Medical treatment is suggested depending on the symptoms.

In this report, not only two interesting siblings with SCKL who have not been diagnosed for 26 years and 14 years, respectively, have been presented, but also SCKL has been reviewed and it has been proposed that there is a spectrum of SCKL that share some common key features, while demonstrating a wide range of phenotypic features. The reason for the female patient to come to our Dermatology clinic was the presence of pigmentary skin changes for a long time. Knowledge about this syndrome, the clinical heterogeneity, complications and the risk of development of malignancy make this disease important for dermatologists.

\section{Conflict of interest}

The authors declare no conflict of interest.

\section{References}

1. Ramalingam K, Kaliyamurthy SD, Govindarajan M, Swathi S. SCKL: a report of a case. J Indian Soc Pedod Prev Dent 2012; 30: 258-61.

2. Thompson E, Pembrey M. SCKL: an overdiagnosed syndrome. J Med Genet 1985; 22: 192-201.

3. Kjaer I, Hansen N, Becktor KB, et al. Craniofacial morphology, dentition, and skeletal maturity in four siblings with SCKL. Cleft Palate Craniofac J 2001; 38: 645-51.

4. Brackeen A, Babb-Tarbox M, Smith J. Pigmentary changes and atopic dermatitis in a patient with SCKL. Pediatr Dermatol 2007; 24: 53-6.

5. Castella M, Pujol R, Callén E, et al. Chromosome fragility in patients with Fanconi anaemia: diagnostic implications and clinical impact. J Med Genet 2011; 48: 242-50.

6. Di Bartolomeo R, Polidori G, Piastra M, et al. Malignant hypertension and cerebral haemorrhage in SCKL. Eur J Pediatr 2003; 162: 860-2.

7. Bobabilla-Morales L, Corona-Rivera A, Corona-Rivera JR, et al. Chromosome instability induced in vitro with mitomycin C in five Seckel syndrome patients. Am J Med Genet 2003; 1A23: 148-52.

8. Arnold SR, Spicer D, Kouseff B, et al. Seckel-like syndrome in three siblings. Pediatr Dev Pathol 1999; 2: 180-7.

9. Guirgis MF, Lam BL, Howard CW. Ocular manifestations of SCKL. Am J Ophthalmol 2001; 132: 596-7.

10. Aktas Z, Yuksel N, Kula S, et al. Chilhood glaucoma as an ophthalmic manifestation of SCKL. J Glaucoma 2013; 22: e3-4.

11. Reddy S, Starr C. SCKL and spontaneously dislocated lenses. J Cataract Refract Surg 2007; 33: 910-2.

12. McIntyre RE, Lakshmnarasimhan Chavali P, Carragher DM, et al. Disruption of mouse Cenpj, a regulator of centriole biogenesis, phenocopies SCKL. PloS Genet 2012; 8: e1003022.

13. Regen A, Nelson LP, Woo SB. Dental manifestations associated with SCKL type II: a case report. Pediatr Dent 2010; 32: 445-50.

14. DeCoster PJ, Verbeeck RHM, Holthaus V, et al. SCKL associated with oligodontia, enamel hypoplasia, delayed eruption, and dentin mineralization: a new variant? J Oral Pathol Med 2006; 35: 639-41.

15. Seymen F, Tuna B, Kayserili H. SCKL: report of a case. J Clin Pediatr Dent 2002; 26: 305-10.

16. SariciD, Akin MA, Kara A, et al. SCKL accompanied by semilobar holoprosencephaly and arthrogryposis. Pediatr Neurol 2012; 46: 189-91.

17. Shanske A, Caride DG, Menasse-Palmer L, et al. Central nervous system anomalies in Seckel syndrome: report of a new family and review of the literature. Am I Med Genet 1997; 70: 155-8.

18. Codd PJ, Scott RM, Smith ER. Seckel syndrome and moyamoya. J Neurosurg Pediatr 2009; 3: 320-4.

19. Thapa R, Muhherjee K. SCKL with asymptomatic tonsillar herniation and congenital mirror movements. J Child Neurol 2010; 25: 231-3.

20. Kumar R, Rawal M, Agarwal S, Gathwala G. Semilobar holoprosencephaly in SCKL. Indian J Pediatr 2008; 75: 519-20.

21. Thapa R, Mallick D, Biswas B, Ghosh A. Open and closed lip schizencephaly in SCKL: a case report. J Child Neurol 2010; 25: 494-6.

22. Adiyaman P, Berberoglu M, Aycan Z, et al. Seckel-like syndrome: a patient with precocious puberty associated with nonclassical congenital abnormality. J Pediatr Endocrinol Metab 2004; 17: 105-10. 
23. Can E, Bulbul A, Uslu S, et al. A case of SCKL with tetralogy of Fallot. Genet Couns 2010; 21: 49-51.

24. Unal Y, Dogan AT, Ozkose Z, Koksal F. Anesthetic management of a patient with SCKL and implanted pacemaker. Paediatr Anaesth 2008; 18: 676-7.

25. Ucar B, Kilic Z, Dinleyici EC, et al. SCKL associated with atrioventricular canal defect: a case report. Clin Dysmorphol 2004; 13: 53-5.

26. Rappen U, von Brenndorff Al. Cardiac symptoms in 2 patients with SCKL. Monatsschr Kinderheilkd 1993; 141: 584-6.

27. Howanietz H, Frisch H, Jedlicka-Köhler I, Steger H. Seckel dwarfism based on a personal case. Klin Padiatr 1989; 201: 139-41.

28. Kutlu R, Alkan A, Kutlu O, Yakinci C. SCKL with polyarteritis nodosa. Indian Pediatr 2004; 41: 1158-61.

29. Deniz K, Kontas O, Akcakus M. Neonatal hepatitis in 2 siblings with Seckel syndrome. Pediatr Dev Pathol 2006; 9 : 81-5.

30. Ozkaya H, Akcan AB, Aydemir G, Kul M. An unusual presentation of Seckel syndrome: fatty liver. Turk J Gastroenterol 2012; 23: 621-3.

31. Hayani A, Suarez CR, Molnar Z, et al. Acute myeloid leukaemia in a patient with Seckel syndrome. J Med Genet 1994; 31: 148-9.

32. Fathizadeh A, Soltani K, Medenica M, Lorincz AL. Pigmentary changes in Seckel's syndrome. I Am Acad Dermatol 1979; 1: 52-4 (abstract). 